EPJ Web of Conferences 82, 01049 (2015)

DOI: $10.1051 /$ epjconf/20158201049

(C) Owned by the authors, published by EDP Sciences, 2015

\title{
Particles separation in a cyclone device cone
}

\author{
Michail Vasilevsky, Pavel Zyatikov ${ }^{\mathrm{a}}$, Lyudmila Shishmina, Pavel Dozmorov, Vera Deeva ${ }^{\mathrm{b}}$ \\ and Yuriy Nikonov
}

National Research Tomsk State University, Lenin Avenue, 36, 634050 Tomsk, Russia

\begin{abstract}
The improvement of the methods for calculating the efficiency for particles separation in these devices is the subject of article. Finding data show that the particles are discharged from the central zone to periphery by diffusive transport in the zone of high centripetal accelerations. It is found that the central zone is the ejection effect zone on the paraxial flow and intense discharge particle zone. The concentrations distribution and distribution of fractional efficiencies are presented.
\end{abstract}

Cyclone devices are a major element in the gas dusting systems in the production of energy, chemicals and other industries. However, the improvement of the methods for calculating the efficiency for particles separation in these devices is the subject of many studies [1] till now. In practice the method for estimating the fractional separation efficiency by integral of the random variable probability is widely accepted [2]. In work [3] it is shown that introduction of dust particles in the wall zone does not guarantee their introduction into the dust collecting bag and theirs 100\% trapping. Ter Linden [4] plotted curves of particles separation equal efficiency. These particles introduced into the various points of the separation volume. It turned out that these curves pass through the point on the axis, i.e. the particles, which introduced below the entry section of the output channel on the axis, trapped with the same efficiency that the particles, which introduced at the periphery. According Kizin [3] the particles, which introduced in the upper axis point of the conical part, trapped more efficiently than the particles introduced into the peripheral zone of the cyclone upper part.

Mentioned data show that the particles are discharged from the central zone to periphery by diffusive transport in the zone of high centripetal accelerations, that is located in the vicinity of radius $\mathrm{Rm}<\mathrm{R} 1$ - the radius of the discharge connection. The vicinity of radius Rm is a transition zone from the quasisolid rotation of the gas to the quasipotential. It is found that the central zone is the ejection effect zone on the paraxial flow and intense discharge particle zone. Turbulence intensity in this zone reaches more than $40 \%$, whereas turbulence intensity in straight channels does not exceed $2 \%$. The arrival process of highly concentrated flow in receiver along the periphery as well as outflow process of low concentration flow from receiver in the paraxial zone is explained by the ejection effects.

In counterflow cyclone in each cone section the flows are directed upwards towards gaz-discharge connection in the paraxial zone and they are directed down towards dust- excretive channel in a

\footnotetext{
a e-mail: zpavel@niipmm.tsu.ru

b e-mail: veradee@mail.ru
}

This is an Open Access article distributed under the terms of the Creative Commons Attribution License 4.0, which permits unrestricted use, distribution, and reproduction in any medium, provided the original work is properly cited. 


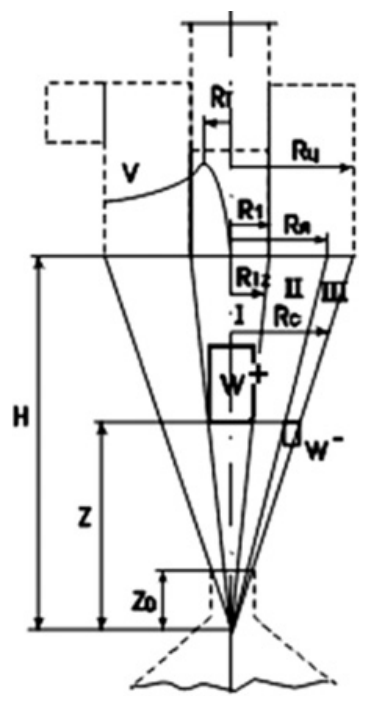

Figure 1. Scheme of the flows into the cyclone.

peripheral zone. The zones of motion flows through the section normal to the axis, are shown schematically in Fig. 1. Zone I - paraxial motion from receiver with attached current from separation space into gaz-excretive flue. Zone II - radial displacement gas zone-core - from peripheral to paraxial zone. Zone III - peripheral zone -the jet stream and the wall layer zone.

In zone I there are subarea of quasi-solid rotational movement and quasipotential flow, in zone II - quasipotential rotation. In the peripheral zone: core boundary $d^{2} \Gamma / d R^{2}=0$; jet stream boundary $d \Gamma / d R=0$; boundary layer $d \Gamma / d R \ll 0$ [5], $\Gamma=R \cdot V, \mathrm{~V}$ - peripheral velocity; $\mathrm{R}$ - actual radius. The total gas mass flow rate through each cross section perpendicular to the cyclone axis is equal zero, i.e. gas consumption in the downstream and upstream flows are equal. The total dust consumption through each section is constant and equal to the amount of dust exuded from the stream in the receiver, or otherwise equal to dust consumption at the inlet of the cyclone multiplied by a purification factor. The last statement follows from the condition of no accumulation of dust in a dedicated cone compartment under the dust flows balance through the surface of the closed compartment contour. To simplify further calculations we consider that the axial velocity in the upstream and downstream flows are distributed evenly along the radius, and downstream flows zone in accordance with the recommendations of [5] in the peripheral region defined by expression $\frac{R_{u}-R_{G}}{R_{u}}=1-r_{G}=0.235+0.07 \cdot b, b-$ width of the upstream end; $\frac{R_{\mathscr{G}}}{R_{u}}=r_{\mathscr{G}}, R_{u}$ - radius of the cyclone cylinder. For high-performance cyclone $1-r_{\mathscr{S}}=0.25 \div 0.26$. From the fig. 1 it follows that cone generators which boundary these zones, have the following meanings: $\frac{R_{1 z}}{R_{u}}=r_{1 z}=r_{1} \frac{Z}{H} ; \quad \frac{R_{y a z}}{R_{u}}=r_{y a z}=r_{G} \frac{z}{H} ; \quad \frac{R_{c z}}{R_{u}}=r_{c z}=\frac{z}{H}, \frac{R}{R_{u}}=r$.

Gas consumption in each section:

$$
Q_{z}=W^{+} \pi R_{u}^{2} r_{1}^{2}\left(\frac{z}{H}\right)^{2}=W^{-} \pi R_{u}^{2}\left(1-r_{Я}^{2}\right)\left(\frac{z}{H}\right)^{2} ; \quad W^{-} / W^{+}=r_{1}^{2} /\left(1-r_{G}^{2}\right)=\text { const. }
$$

Dust consumption of particular fraction in each section:

$$
G_{z}=Q_{B x} C_{B x} \eta_{\delta}=W^{+} \pi r_{1}^{2} R_{u}^{2} C_{B x} \eta_{\delta}=2 \pi W^{-} \int_{R Я Я A}^{R c z} C R \cdot d R-2 \pi W^{+} \int_{0}^{R_{1} z} C R \cdot d R .
$$


Dividing the left and right parts (1) into $\pi R_{u}^{2} \cdot U_{u} C_{B x}=Q_{B x} C_{B x}$, we obtain

$$
\eta_{\delta}=\frac{2}{1-r_{Я}^{2}} \cdot \int_{r_{c z}}^{r_{c z}} c r d r-\frac{2}{r_{1}^{2}} \int_{0}^{r_{1 z}} c r d r .
$$

In these equations $\eta$ - collection efficiency of particles size $\delta$; $C_{B x}$ - the concentration of particles size $\delta$ in gas; c - actual concentration of particles size $\delta$ in gas; $Q$ - gas consumption.

We consider that the presence of the does not affect the gas, $W^{-}=$const; $W^{+}=$const; index of power $\mathrm{n}$ of the peripheral velocity distribution in the quasi-potential zone has the same value in the different sections, particles transport in a radial direction is estimated by particles flow in their averaged relative motion when centrifugal forces and turbulent diffusive flow influence these particles. The transfer equation in a radial direction has image

$$
\left.\mathrm{C} \Delta \mathrm{u}\right|_{z}=\left.\varepsilon \frac{\mathrm{dC}}{\mathrm{dr}}\right|_{z}
$$

$\Delta u=\frac{V^{2}}{R} \tau, \tau=\frac{\delta^{2} \rho_{\delta}}{18 \nu 8}, \quad \Delta \overline{\mathrm{u}}=\frac{\Delta \mathrm{u}}{U_{\mathrm{L}}} ; \quad v_{B x}=\frac{V_{B x}}{U_{u}}, \quad \Delta \overline{\mathrm{u}}=k \frac{v^{2}}{\mathrm{r}} \mathrm{Stk} ; \quad S t k=\frac{U_{u} \tau}{R_{u}}, \quad v=\frac{v B_{x} \mathrm{r}}{\mathrm{r}_{T}^{n+1}} \quad$ when $\quad r<$ $r_{T}, v=\frac{v B_{x}}{\mathrm{r}^{n}}$ when $r>r_{T} ; \quad U_{u}=\frac{Q}{\pi R_{u}^{2}}$. Where $Q-$ gas consumption in the cyclone, $\mathrm{R}_{u}$ - radius of the cyclone; $\delta$ - particle diameter; $\rho_{\delta}, \rho$ - particles and gas density, $v$ - kinematic viscosity coefficient; $n$-index in the equation $v \cdot r^{n}=$ const $=v_{B X} \cdot 1^{n} ; k<1$ - coefficient taking into account the resistance increase when deviation from the Stokes flow under the fluctuations effect [6]. Index $n$ changes height over the range $0,2-0,7[7]$.

To simplify the calculation we consider $\Delta \bar{u}=\mathrm{Stk} \cdot \mathrm{A} / \mathrm{r}$, where $\mathrm{A}$ is found from the relation $\int_{r_{*}}^{1} \frac{A}{r} r \cdot d r=\int_{0}^{1} \frac{v^{2}}{r} r \cdot d r \Rightarrow A=\frac{\int_{0}^{1} v^{2} \cdot d r}{1-r_{*}}$; index $r_{*}$ is chosen from the condition $\int_{0}^{r_{*}} v^{2} d r=\int_{r_{*}}^{r_{T}} v^{2} d r$. For the section $\mathrm{z}: \mathrm{r}_{*_{z}}=\mathrm{r}_{*} \cdot \frac{Z}{H} ; \mathrm{r}_{T z}=\mathrm{r}_{\mathrm{T}} \cdot \frac{\mathrm{Z}}{\mathrm{H}}$. Then $A_{z}=v_{B x}^{2}\left(\frac{H}{z}\right)^{2 n} \frac{3-r_{T}^{1-2 n}(n+1)}{3(1-2 n)\left(1-r_{*}\right)}, \varepsilon=\frac{V B_{B x} \cdot a \cdot b}{2 \pi H(n+1)}$.

Where $\mathrm{a}, \mathrm{b}$ - height and width of the upstream end. We denote $\alpha=\frac{\Delta \mathrm{u}_{z} \mathrm{R}_{u}{ }^{*}}{\varepsilon}=\frac{\Delta \bar{u} 2 \pi \bar{h}(n+1)}{{ }^{v} B_{x} \cdot \bar{\alpha} \cdot \bar{b}}$. we can write transport equation (3) in dimensionless form $\frac{c \alpha_{z}}{r}=\frac{d c}{d r}$ when $r>r_{*_{z}}, c_{z}=c_{0 z}$ when $r \leq r_{*_{z}}$

$$
c_{z}=c_{0 z}\left(\frac{r}{r_{* z}}\right)^{\alpha_{z}} .
$$

Calculation shows that in expression (3) $\int_{0}^{r_{1}(z)} C r \cdot d r=C_{0 z}\left[\frac{r_{*}^{2}}{2}+\frac{r_{1}^{\alpha_{z}+2}-r_{*}^{\alpha_{z}+2}}{r_{*}\left(\alpha_{z}+2\right)}\right]\left(\frac{z}{H}\right)^{2}$, and taking into account (5):

$$
\frac{\mathrm{C}_{0 z}}{\mathrm{C} \theta x}=\frac{\left(\frac{\mathrm{H}}{\mathrm{Z}}\right)^{2} \eta_{\delta} \mathrm{r}_{*}{ }^{\alpha_{z}}\left(\alpha_{z}+2\right)}{\frac{1-\mathrm{r}_{\mathscr{G}}^{(\alpha+2)}}{1-\mathrm{r}_{\mathscr{G}}^{2}}-\frac{1}{2 \mathrm{r}_{1}^{2}}\left(2 \mathrm{r}_{1}^{\alpha_{z}+2}+\alpha_{z} \mathrm{r}_{*}^{\left(\alpha_{z}+2\right)}\right)} .
$$

Thus, the concentrations distribution by the ratio (5) taking into account (6) can be represented for example for cyclone SC-CF-34 when $\rho_{\delta} / \rho=2000 ; \mathrm{R}_{u}=0.15 \mathrm{~m} ; U_{u}=1.7 \frac{\mathrm{m}}{\mathrm{c}} ; \mathrm{v}=1.5 \cdot 10^{-5} \frac{\mathrm{m}^{2}}{\mathrm{c}}$; $h_{k}=4 ; r_{1}=0.34 ; \bar{a} \cdot \bar{b}=1.3 \cdot 0.43 ;$ and $\bar{r}_{*}=0.6 \cdot r_{1} ; r_{T}=0.8 \cdot r_{1} ; n_{c p}=0.55 ; h=H / / R_{u}{ }^{c}=$ 5,$61 ; k=1$ and distribution of fractional efficiencies [2].

Table 1.

$$
\begin{array}{cccc}
\delta(\mathrm{mcm}) & 1 & 3 & 5 \\
\eta & 0.41 & 0.82 & 0.91
\end{array}
$$

at the cyclone upper part the relative concentration of particle size $3 \mathrm{mcm}$ is equal 0,41 on the axis and 1,5 at the periphery, and at dust-excretive holes zone is equal 0,37 and 36 correspondingly. 


\section{EPJ Web of Conferences}

This work was supported by as part of the program to improve the competitiveness of the Tomsk Polytechnic University.

\section{References}

[1] M.V. Vasilevsky, E.G. Zykov, A.S. Razva, The calculation model of particles concentration in a counterflow cylindrical cyclone device. Theoret. chemical bases. technology, 45, No 3, 7 (2011)

[2] Ed. A.A. Rusanov, Directory on dust and ash collection (Energy, Moscow, 1983)

[3] M.G. Kizin, Calculation methods and recommendations for gas cyclone devices (Vladimir Research Institute of synthetic resins, Vladimir, 1970)

[4] A.J. Ter Linden, Cyclone Dust Collectors for boilers, Trans. ASME. 75, (1953)

[5] A.N. Shtym, Aerodynamics of cyclone-vortex chambers (Far Eastern Univ., Vladivostok, 1985)

[6] E.P. Mednikov, Turbulent transport and deposition of aerosols (Scienc, Moscow, 2009)

[7] M.V. Vasilevsky, The gas dedusting by inertial devices (Publ. House of the Tomsk Polytech. Univer, Tomsk, 2008) 